\title{
From Denial of Facts to Rationalization and Avoidance: Ideology, Needs, and Gender Predict the Spectrum of Climate Denial and Self-Protection
}

\author{
Marlis Charlotte Wullenkord
}

Social, Environmental, and Economic Psychology, Department of Psychology, University of Koblenz-Landau, Fortstraße 7, 76829 Landau, Germany

E-Mails:wullenkord@uni-landau.de

Correspondence regarding this manuscript should be sent to: Marlis Wullenkord, Department of Psychology, University Koblenz-Landau, Fortstraße 7, 76829 Landau, Germany wullenkord@uni-landau.de

\section{Other Information}

Funding: Marlis Wullenkord was supported by a PhD-scholarship by the German Federal Environmental Foundation (Deutsche Bundesstiftung Umwelt, DBU). The DBU had no involvement in study design, in the collection, analysis and interpretation of data, in the writing of the report, and in the decision to submit the article for publication.

Data availability statement: Original data presented in this article are available on request. 
IDEOLOGY, NEEDS, GENDER, AND THE SPECTRUM OF DENIAL

\section{From Denial of Facts to Rationalization and Avoidance: Ideology, Needs, and Gender Predict the Spectrum of Climate Denial and Self-Protection}

Denying climate change can take many forms, ranging on a spectrum from outright denial of facts (literal denial), over re-interpretation or distortion of facts (interpretive denial, e.g., denial of personal and global outcome severity), to the acknowledgement of facts but denial of their implications (implicatory denial, e.g., avoidance, denial of guilt, rationalization of own involvement). This study aimed at 1) exploring potential distinct profiles within the spectrum of climate denial and self-protection and 2) investigating relations with right-wing ideological conviction and gender (established predictors) but also need satisfaction and value orientation. Analysis of a German quota sample $(N=1007)$ revealed the following: Participants differed in the extent to which they endorsed all types of climate-denial and self-protection but there were no distinct profiles of climate denial and self-protection in the data, according to latent profile analysis. As pre-registered, structural equation modelling revealed that people who reported right-wing ideological convictions reported more climate denial and selfprotection along the spectrum of denial but especially literal and interpretive denial. Absence of need satisfaction and male gender were significant additional but weaker predictors of implicatory denial. Future research should employ longitudinal, experimental, mixed-methods designs to further disentangle the underlying mechanisms and functionality of climate denial.

Keywords: climate change; psychological needs; climate denial; defensiveness; selfprotection; ideology; gender 


\section{Introduction}

"Not our climate is in jeopardy - our freedom is"

- slogan of EIKE (European Institute for Climate and Energy) ${ }^{1}$, Germany's biggest lobby for systematic climate denial

It is an established finding that right-wing ideological conviction and male gender predict agreement with such statements (e.g., Feygina et al., 2010; Jylhä et al., 2016;

McCright \& Dunlap, 2011; Milfont et al., 2021). Most studies thus far have considered climate denial to be relatively literal - the outright denial of the fact that the Earth's climate is warming due to anthropogenic emission of carbon into the atmosphere. However, climate denial is more than outright denial of facts. It exists on a spectrum, ranging from literal denial over interpretive denial (i.e., the re-interpretation or distortion of facts) to implicatory denial (i.e., the acknowledgement of facts and denial of their implications; Cohen, 2001; Norgaard, 2019). From a psychological perspective, it fulfills the function of protecting the self from threat and uncomfortable emotions (Cohen, 2001; Norgaard, 2006). This research aims at investigating the spectrum of climate denial and self-protection more closely, explores potential distinct profiles within it, and investigates relations with right-wing ideological conviction and gender but also need satisfaction and value orientation.

\subsection{Climate Denial and Self-Protection on a Spectrum}

Norgaard (2019) describes a political spectrum of climate denial, ranging from literal forms of outright denial of the facts on the right to implicatory denial on the left. While the manifested shape of the denial is different, the function it serves is similar across the spectrum: It is a reaction to (psychological) threat and ultimately entails of a reinforcement of social structures and solidification of power relations - a means to protect the self from threat (Norgaard, 2006). Similarly, Stoll-Kleemann and O'Riordan (2020) found that more implicatory forms of denial (here denial of responsibility) replaced more literal forms of

\footnotetext{
${ }^{1}$ https://www.eike-klima-energie.eu/, last accessed 05/07/2021
} 
denial in a large German sample over time. The Climate Self-Protection scale (Wullenkord \& Reese, 2020) measures different forms of climate denial and self-protection. This validation study suggested that people use strategies such as denial of the global and personal consequences of climate change (interpretive denial), rationalization of one's own involvement (similar to denial of responsibility), denial of guilt, and avoidance of the problem (implicatory denial). In line with predictions that denial leads to inactivity (Cohen, 2001), those strategies were negatively associated with environmental outcomes, such as environmental awareness, autonomous motivation to protect the environment, and selfreported pro-environmental behavior (see also Homburg et al., 2007; Ojala, 2012).

In the present study, I assessed climate denial and self-protection along the spectrum of denial using a German quota sample (see right side of Figure 1 for operationalization) and explored whether there were distinct profiles of climate denial and self-protection in the data. As pre-registered ${ }^{2}$, I hypothesized that:

H1: Climate denial and self-protection are negatively associated with policy support and pro-environmental intentions.

\subsubsection{Right-Wing Ideological Conviction and Male Gender}

Most studies investigating climate denial focus on denial in its literal and interpretive sense, without explicitly distinguishing between different forms of denial. Right-wing political conviction has been identified as a major predictor of literal climate denial (e.g., Feygina et al., 2010; Jylhä et al., 2016; McCright \& Dunlap, 2011; Milfont et al., 2021). Most studies considered the following indicators: 1) Right-wing authoritarianism: The adherence to conservative norms and values, a preference for obedience to authorities, strict laws, and harsh punishments (RWA; Altemeyer, 1981; Stanley et al., 2017); 2) Social dominance orientation: The preference for group-based social hierarchies and support for dominance and

\footnotetext{
${ }^{2}$ All hypotheses were pre-registered at https://aspredicted.org/blind.php? $\mathrm{x}=\mathrm{js} 5 \mathrm{pc} 4$
} 
Ideology, NeEds, Gender, AND the SPectrum of Denial

devaluation of low-status groups (SDO; Jylhä et al., 2016; Pratto et al., 1994); 3) Human dominance over nature: The belief that humans are distant from and superior to nature and have the right to control it for their own benefit (ND, Jylhä \& Akrami, 2015; Milfont et al., 2013); 4) System justification: The desire to rationalize, legitimize, and defend the status quo (Feygina et al., 2010; Jost \& Banaji, 1994); and 5) Political left-right orientation (Norgaard, 2019). In the present study, I assess the combined and unique contributions of these indicators of right-wing ideological conviction to investigate their general relations with different forms of climate denial and self-protection along the spectrum of denial. Another consistent predictor of climate denial and self-protection is male gender (Hultman \& Pulé, 2018; Jylhä et al., 2016; McCright \& Dunlap, 2011; Nelson, 2020). Climate denial may be an expression of system-justifying ideologies that serve as a protection of male privilege (see Jylhä et al., 2016). I therefore hypothesized that:

H2: Right-wing ideological conviction is positively associated with climate denial and self-protection, especially literal and interpretive denial.

H3: Male gender predicts climate denial and self-protection, especially literal and interpretive denial.

H4: Right-wing ideological conviction mediates the association between gender and climate denial and self-protection.

\subsubsection{Basic Psychological Need Satisfaction and Relative Value Orientation}

The literature on implicatory denial is less straightforward. Ideological conviction may also explain the use of implicatory denial but associations are likely weaker than for literal and interpretive denial (see Norgaard, 2019). This warrants considering a more fundamental indicator of defensiveness and human functioning, such as the satisfaction of basic psychological needs (henceforth needs) as proposed in self-determination theory (SDT, Ryan $\&$ Deci, 2017). SDT as a humanistic, dialectical theory of human motivation assumes that humans become defensive and self-protect if their needs for autonomy (agency), competence 
Ideology, NeEds, Gender, AND the SPectrum of Denial

(efficacy), and relatedness (belongingness) are frustrated (Vansteenkiste \& Ryan, 2013). It is theoretically feasible to apply it to human responses to the climate crisis (Wullenkord, 2020). Various studies show that need satisfaction is associated with pro-environmentalism (e.g., Cooke et al., 2016; Kaplan \& Madjar, 2015; Tröger et al., 2021), and there is first empirical evidence showing that absence of need satisfaction in fact predicts both interpretive and implicatory climate denial and self-protection (M. C. Wullenkord, under review).

One influence on need satisfaction is the endorsement of values. According to goal contents theory (Kasser \& Ryan, 1996; Ryan \& Deci, 2017), the pursuit of intrinsic values that "are expressive of desires congruent with actualizing and growth tendencies natural to humans" (e.g., community feeling, affiliation, self-acceptance, Kasser \& Ryan, 1996, p. 280) satisfies needs. The pursuit of extrinsic values that "depend on the contingent reactions of others (and usually serve) as means to another end" (e.g., financial success, popularity, image, Kasser \& Ryan, 1996, p. 280), however, frustrates needs. All humans strive for both intrinsic and extrinsic values but differ in the extent to which they endorse them. Given that industrial capitalism promotes a relatively extrinsic value orientation (Kasser \& Ryan, 1996), people with a relatively extrinsic value orientation may try to defend the system and thus deny climate change. I hypothesized that:

H5: Need satisfaction is negatively associated with climate denial and self-protection. H6: Relative intrinsic value orientation is negatively associated with climate denial and self-protection, mediated by need satisfaction.

H7: Relative extrinsic value orientation is positively associated with need frustration and right-wing ideological conviction.

\subsection{The Present Study and Analytical Approach}

In previous studies investigating climate denial and self-protection and their relations with different indicators of environmentalism, some findings were contradictory (e.g., 
regarding denial of guilt, Wullenkord \& Reese, 2020). Furthermore, effect sizes were small, indicating unobserved variance in the results. This unexplained heterogeneity may indicate that some self-protective strategies may in fact be healthier and more proactive strategies than others, not hindering pro-environmentalism from occurring. One possible explanation for higher unobserved variance may be the existence of latent subgroups in the population, yielding different environmental relations depending on subgroup belongingness (i.e., different profiles of climate denial and self-protection may be more or less adaptive and may thus be associated with more or less pro-environmentalism). However, environmental psychology primarily employs variable-centered analytical approaches. These are limited because they cannot analyze the data for the existence of latent subgroups, often become disproportionately complex, and require disproportionately high power if all relevant interaction terms are included in traditional models, such as moderated regression analysis. The present study thus combines variable- and person-centered approaches: Firstly, it aimed at exploring whether distinct profiles of climate denial and self-protection could be identified in a German quota sample (person-centered approach), using latent profile analysis (LPA, Collins \& Lanza, 2010). Secondly, it aimed at investigating how different forms of climate denial and self-protection relate to need satisfaction, value orientation, ideological conviction, and gender. This is a variable centered approach. To my knowledge, this is the first study that combines person- and variable-centered analytic approaches to investigate climate denial and self-protection, its psychological predictors, and pro-environmental outcomes in a German quota sample. 


\section{Methods}

\subsection{Participants and Procedure}

A German quota sample ( $N=1134$, stratified for age and gender, analyzed sample size after data cleaning ${ }^{3} N=1007$ ) participated in an online study hosted on the platform SoSciSurvey (Leiner, 2020). Participants were recruited through the online-access panel provider Respondi AG. Age ranged from 18 to $69(M=43.91, S D=13.97)$ and 51.14\% of the participants were female. Educational level was slightly above average: $26.31 \%$ had university entrance qualification and $29.48 \%$ had a university degree, and on average participants reported a monthly income between 1500 and $2500 €$. After giving their informed consent, participants answered questions about six types of climate denial and self-protection (avoidance, denial of guilt, rationalization of own involvement, denial of personal outcome severity, denial of global outcome severity, literal denial), ideological conviction (RWA, SDO, ND, system justification, political orientation), need satisfaction, value orientation, proenvironmental intentions, policy support, and socio-demographic variables (age, gender, education, income). Materials used are described in detail in supplementary material S1. Further variables and preparation of the raw data are detailed in Wullenkord et al. (2021), where we focused on analyzing climate anxiety and its correlates. The local ethics committee granted ethical approval for the study in line with the DGPS and Helsinki declarations (293_2020).

\subsection{Statistical Analysis}

I used R, version 4.0.3 (R Core Team, 2020) for all analyses: First, I performed an LPA to explore the data for discrete profiles of climate denial and self-protection. LPA is large sample method. Based on Monte-Carlo simulations, Tein et al. (2013) suggest that for

\footnotetext{
${ }^{3}$ See details in supplementary materials S2 and S3.
} 
IDEOLOGY, NeEdS, GENDER, AND THE SPECTRUM OF DENIAL

an LPA with Cohen's $d=.8$ and 10 indicators of class membership, sample size should be at least 500 (see Nylund et al., 2007 for similar recommendations). I therefore aimed for recruiting at least $N=1000$ participants to perform analyses with sufficient power. This data set was large enough to randomly split it in half and to replicate and thus validate the solution in the second half of the data. Second, I used structural equation modelling to investigate variable-centered hypotheses. Please refer to supplementary materials S2 and S3 for detailed results of the whole data analysis both with (S2) and without exclusion of outliers (S3).

\section{Results}

See Table 1 for descriptive statistics and correlations of study variables. Most people reported medium-low levels of climate denial and self-protection, slightly higher levels of right-wing ideology, stronger need satisfaction than frustration, relatively intrinsic value orientation, medium pro-environmental intentions, and high policy support. Correlation analyses revealed that need satisfaction was weakly negatively related to most types of climate denial and self-protection (there was only a trend for denial of global outcome severity and a positive relation with denial of guilt). There were weak to medium associations between relative extrinsic value orientation and all types of climate denial and self-protection (except for denial of guilt, which was unrelated) and ideology (except for system justification, which was unrelated). All indicators of right-wing ideology (except for system justification, which was unrelated or negatively related) were medium positively related to all types of climate denial and self-protection. Male gender was weak to medium positively related to all types of climate denial and self-protection (except for avoidance, which was unrelated). Finally, all types of climate denial and self-protection were medium negatively related to proenvironmental intentions and policy support. 
IDEOLOGY, NEEDS, GENDER, AND THE SPECTRUM OF DENIAL

Table 1

$0 \quad$ Spearman correlations and descriptive statistics of study variables

\begin{tabular}{|c|c|c|c|c|c|c|c|c|c|c|c|c|c|c|}
\hline Variable & 1 & 2 & 3 & 4 & 5 & 6 & 7 & 8 & 9 & 10 & 11 & 12 & $M$ & $S D$ \\
\hline 1) Avoidance & .89 & & & & & & & & & & & & 3.04 & 1.18 \\
\hline 2) Denial of guilt & $.10^{* *}$ & .83 & & & & & & & & & & & 3.92 & 1.39 \\
\hline 3) Rationlization of own involvement & $.44 * *$ & $.57 * *$ & .92 & & & & & & & & & & 3.18 & 1.46 \\
\hline 4) Denial of personal outcome severity & $.45^{* *}$ & $.46^{* *}$ & $.61 * *$ & .87 & & & & & & & & & 2.35 & 1.21 \\
\hline 5) Denial of global outcome severity & $.42 * *$ & $.57 * *$ & $.65^{* *}$ & $.71 * *$ & .91 & & & & & & & & 2.58 & 1.59 \\
\hline 6) Literal denial & $.37 * *$ & $.53 * *$ & $.61 * *$ & $.63 * *$ & $.83 * *$ & .94 & & & & & & & 2.45 & 1.55 \\
\hline 7) Basic psychological need satisfaction & $-.19 * *$ & $.10 * *$ & $-.08 *$ & $-.07 *$ & $-.06^{\dagger}$ & $-.06^{*}$ & .87 & & & & & & 5.06 & 0.87 \\
\hline 8) Relative extrinsic value orientation & $.21 * *$ & .02 & $.19 * *$ & $.19 * *$ & $.15 * *$ & $.12 * *$ & $-.24 * *$ & .89 & & & & & -1.26 & 0.95 \\
\hline 9) Right-wing authoritarianism & $.27 * *$ & $.33 * *$ & $.32 * *$ & $.29 * *$ & $.38 * *$ & $.38 * *$ & .04 & $.19 * *$ & .86 & & & & 3.90 & 1.32 \\
\hline 10) Social dominance orientation & $.21 * *$ & $.20 * *$ & $.30 * *$ & $.34 * *$ & $.34 * *$ & $.32 * *$ & -.05 & $.22 * *$ & $.35 * *$ & .75 & & & 2.45 & 1.00 \\
\hline 11) Human dominance over nature & $.25 * *$ & $.22 * *$ & $.33 * *$ & $.37 * *$ & $.37 * *$ & $.34 * *$ & -.03 & $.17 * *$ & $.27 * *$ & $.32 * *$ & .88 & & 2.64 & 1.24 \\
\hline 12) System justification & .00 & $-.15 * *$ & $-.13 * *$ & .02 & $-.10 * *$ & $-.12 * *$ & $.17 * *$ & -.04 & -.06 & $.15^{* *}$ & $.12 *$ & .82 & 3.98 & 1.16 \\
\hline 13) Gender ( $1=$ female $)$ & .00 & $-.14 * *$ & $-.22 * *$ & $-.11 * *$ & $-.12 * *$ & $-.09 * *$ & -.04 & $-.09 *$ & $-.08 *$ & $-.07 *$ & $-.15^{* *}$ & .04 & & \\
\hline
\end{tabular}

1 Note. ${ }^{\dagger} p<.10, * p<.05, * * p<.01$ 
IDEOlogy, NeEds, Gender, AND the SpeCtrum of Denial

\subsection{Profiles of Climate Denial and Self-Protection}

I used the R-packages mclust (Scrucca et al., 2016) and tidyLPA (Rosenberg et al., 2018) to perform an LPA that analyzed whether there were discrete profiles of climate denial and self-protection in the sample. LPA is a probability-based mixture modelling approach aiming at identifying latent profiles within large, heterogeneous populations on the basis of observed continuous indicator variables (Collins \& Lanza, 2010). The identified profiles have different configurations, which are either qualitatively different (i.e., difference in shape) or quantitatively different (i.e., difference in levels), and properties in relation to both antecedents and outcome variables.

The six types of climate denial and self-protection served as indicators. There were no missing data and multivariate outliers were removed based on Mahalanobis distance with a cutoff of $p<.001$ when cleaning the data (see supplementary material S2), as multivariate outliers can distort the results of LPA when extreme cases inform profiles. Finally, I randomly split the data set in half, with the first half of the data set comprised $n_{1}=515$ cases and the second half comprising $n_{2}=496$ cases.

I estimated solutions comprising one to nine different profiles (see supplementary materials for model estimations and visualizations). A solution with nine profiles had best fit indices (Tein et al., 2013), Bayesian information criterion $(B I C)=6999.41$, Sample sizeadjusted $\mathrm{BIC}=6783.57$, Akaike information criterion $(\mathrm{AIC})=6710.94$. Bootstrapped sequential likelihood ratio test (McLachlan \& Peel, 2000) for the number of mixture components indicated a six profile solution to perform significantly better than a seven profile solution, BLRT $=52.03, p<.001$. Inspection of the elbow point (Nylund-Gibson \& Choi, 2018) suggested a solution with four profiles to be most feasible. These results were replicated using the second half of the data. Nevertheless, none of the configurations revealed discrete profiles but rather separated participants based on levels of responses to the indicator variables:

Profile 1 was low on all types of climate denial and self-protection, higher profiles were 
IDEOLOGY, NeEdS, GENDER, AND THE SPECTRUM OF DENIAL

higher on all types of climate denial and protection, and there was significant overlap between profiles. This information can also be deduced from correlation analyses: All types of climate denial and self-protection had relatively high inter-correlations, rendering a variable-centered analytical approach was deemed more appropriate for further analyses.

\subsection{Structural Equation Model Predicting Climate Denial and Self-Protection}

I employed the R-package lavaan, version 0.6-3 (Rosseel, 2012) to estimate a structural equation model predicting climate denial and self-protection.

\subsubsection{Hypothesized Model and Assumptions}

Given multivariate non-normal distribution of variables, $E(14)=1540.76, p<.001$, I used robust maximum likelihood estimation with Satorra-Bentler correction (Finney \& DiStefano, 2013) for model estimation. As pre-registered, I hypothesized paths from need satisfaction, value orientation, right-wing ideology, and gender to all types of climate denial and self-protection. Furthermore, I estimated paths from gender to ideology, from ideology to value orientation, and from value orientation to need satisfaction. See Figure 1 for a visualization of the model with significant path coefficients and see supplementary materials for standardized residuals and empirical and model-implicated variance-covariance-matrices.

I defined a latent variable for ideology using mean scores of RWA, SDO, ND, and system justification ${ }^{4}$ as indicator variables and a latent variable for need satisfaction using mean scores of relatedness, autonomy, and competence satisfaction as indicator variables. The analysis was well-powered, with $N=1007$ and 62 free parameters, $N / q=16.51$. The model was identified.

\footnotetext{
${ }^{4}$ Given that some items assessing ideological beliefs had strong overlap between constructs, I performed an exploratory main axis analysis over all items assessing ideology (i.e., items comprising the RWA, SDO, ND, and system justification measures, and political orientation). Horn's parallel analysis yielded four clearly interpretable factors after exclusion of cross-loading items and items with factor loadings $<.3$ (see supplementary materials S2 and S3).
} 


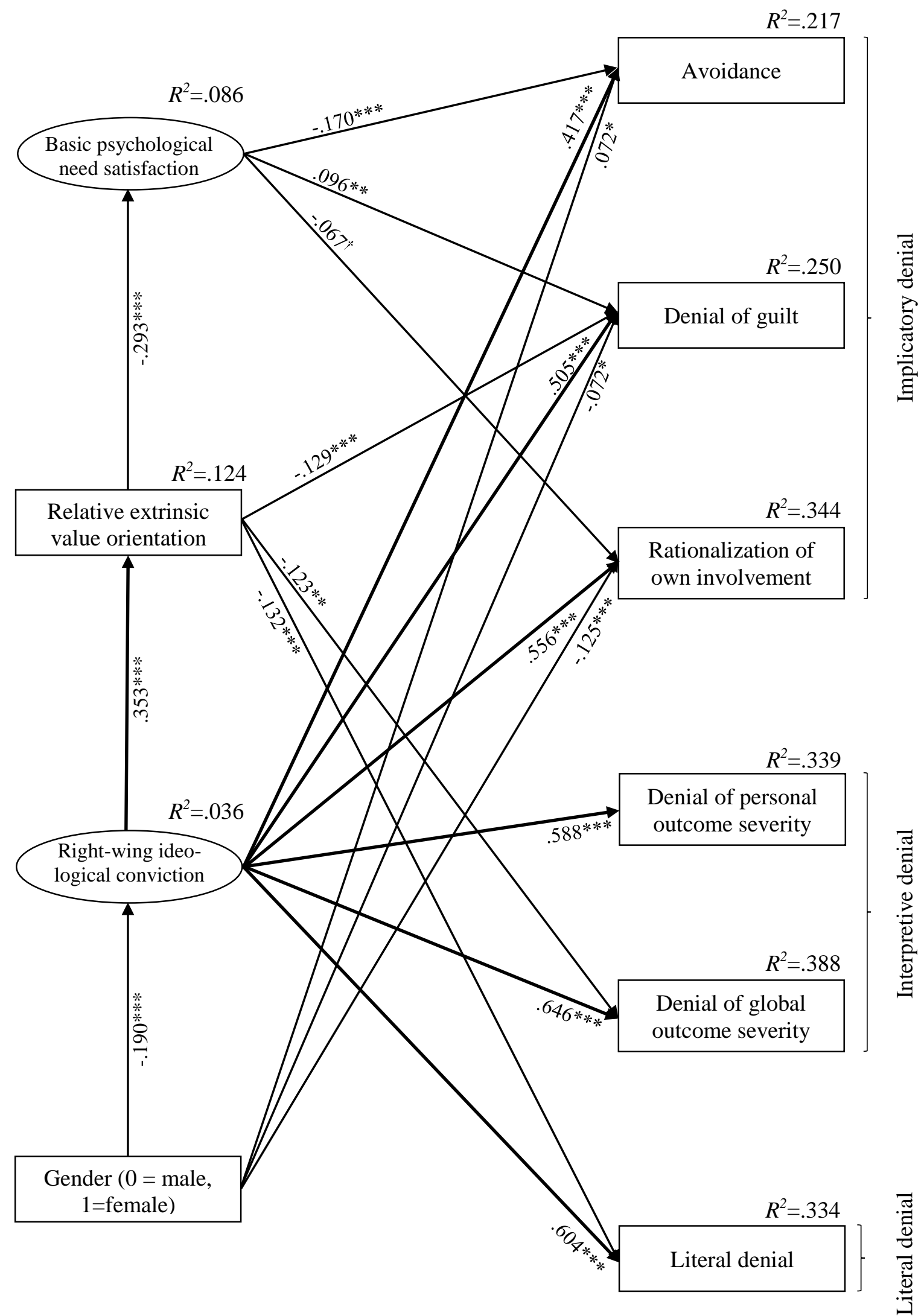

Figure 1. Structural equation model predicting climate denial and self-protection from basic psychological need satisfaction, value orientation, ideological conviction, and gender. Displayed are standardized parameter estimates for significant paths. Thicker paths represent stronger relations. ${ }^{\dagger} p<.10, * p<.05, * * p<.01, * * * p<.001$ 
IDEOLOGy, NeEdS, Gender, AND the SPECTRum Of DENIAL

\subsubsection{Model Estimation}

Fit indicators indicated good fit of the hypothesized model to the data (Hu \& Bentler, 1999): Satorra-Bentler $\chi^{2}(56, N=1007)=267.09, p<.001$, Robust Comparative Fit Index $(\mathrm{CFI})=.957, \mathrm{AIC}=40162.46$, Robust Root Mean Square Error of Approximation $($ RMSEA $)=.066,90 \%$ CI[.058, .074], Standardized Root Mean Square Residual $($ SRMSR $)=.051$. Because the latent ideology variable explained no variance in system justification $\left(R^{2}=.001\right)$, I estimated another model without system justification. The adapted model fit the data well: Satorra-Bentler $\chi^{2}(43, N=1007)=123.66, p<.001$, Robust CFI=.983, $\mathrm{AIC}=37000.43$, Robust RMSEA $=.046,90 \% \mathrm{CI}[.037, .056], \mathrm{SRMSR}=.035$. It had better fit than the hypothesized model, $\chi^{2} \operatorname{diff}(13, N=1007)=140.40, p<.001$. Need satisfaction negatively predicted avoidance, and rationalization of own involvement, and positively predicted denial of guilt; surprisingly relative intrinsic value orientation predicted denial of guilt, denial of global outcome severity, and literal denial; right-wing ideology predicted all types of climate denial and self-protection; male gender predicted denial of guilt and rationalization, and female gender predicted avoidance. Male gender predicted right-wing ideological conviction, right-wing ideological conviction predicted relative extrinsic value orientation, and relative intrinsic value orientation predicted need satisfaction.

\section{Discussion}

This the first study that considers a spectrum of climate denial and self-protection and relates it to a range of predictors, namely ideological conviction, need satisfaction, value orientation, and gender. Participants differed in the extent to which they endorsed all types of climate-denial and self-protection but there were no distinct profiles of climate denial and self-protection in the data. People who reported right-wing ideological conviction also reported more climate denial and self-protection along the spectrum of denial but especially 
Ideology, NeEds, Gender, AND the SPectrum of Denial

literal and interpretive denial. Need satisfaction and gender were significant additional but weaker predictors of implicatory denial: People whose needs were less satisfied reported more avoidance and rationalization of their own involvement but less denial of guilt. Men reported more denial of guilt and rationalization of their own involvement, while women reported more avoidance.

These findings are only partly in line with the idea of a political spectrum of denial (Norgaard, 2019). Here, right-wing ideological conviction predicted all types of climate denial and self-protection, even if it was a stronger predictor for literal and interpretive denial. Nevertheless, most research on climate denial has been conducted in the US and even though part of the Global North, may not generalize to Germany (Hornsey et al., 2018). Furthermore, right-wing ideological conviction as operationalized in this study may differ from identifying as right-wing on a simple left-right spectrum.

Notably, system justification was uncorrelated with RWA and only weakly related to SDO and ND. Even across the Global North, there are vast differences in how just systems are and how normalized it is to hold certain (political) views. In Germany, living standards tend to be relatively high for all people. System justification as assessed in this study may not be an ideological position reflecting meritocratic beliefs and a right-wing ideology serving to maintain one's own privilege. Instead, it may be a more or less rational estimation of the system as providing a social safety net for most and averting major social injustices. Findings that system justification is a weaker predictor of climate denial in the Swedish context (Jylhä \& Akrami, 2015) and that it was uncorrelated with RWA (in contrast to findings in the US context, Feygina et al., 2010) may mirror this interpretation. In line, the items used in this study may not have captured the underlying ideology justifying the power structures industrial capitalism produces and maintains (in contrast to items measuring SDO), along with all the associated consequences for (climate) justice. 
IDEOlogy, NeEds, Gender, AND the SPECtrum of Denial

\subsection{Limitations and Future Directions}

The present study employed a large quota sample and is the first to investigate a spectrum of climate denial and self-protection, thereby extending both previous research that has relied on non-representative populations or did not differentiate different forms of climate denial and self-protection. Nevertheless, this study was cross-sectional and thus only gives a glimpse into the functionality and underlying mechanisms of climate denial and selfprotection. Longitudinal and experimental designs are warranted to further understand the "why" and "how" of climate denial and self-protection. A possible approach could be to implement need-based interventions targeting the different profiles of denial. These could aim at disentangling further what needs are met with right-wing ideological convictions and consequent climate denial and self-protection, reducing climate denial and self-protection, and increasing pro-environmental action. For instance, need-based communication is less threatening because needs are being satisfied, providing people with psychological resources to face information that is difficult in itself. 
IDEOLOGY, NeEdS, GENDER, AND THE SPECTRUM OF DENIAL

\section{References}

Altemeyer, B. (1981). Right-Wing Authoritarianism. University of Manitoba Press.

Cohen, S. (2001). States of Denial: Knowing About Atrocities and Suffering. Polity Press.

Collins, L. M., \& Lanza, S. T. (2010). Latent Class and Latent Transition Analysis. 332.

Cooke, A. N., Fielding, K. S., \& Louis, W. R. (2016). Environmentally active people: The role of autonomy, relatedness, competence and self-determined motivation. Environmental Education Research, 22(5), 631-657. https://doi.org/10.1080/13504622.2015.1054262

Feygina, I., Jost, J. T., \& Goldsmith, R. E. (2010). System justification, the denial of global warming, and the possibility of "system-sanctioned change." Personality and Social Psychology Bulletin, 36(3), 326-338. https://doi.org/10.1177/0146167209351435

Finney, S. J., \& DiStefano, C. (2013). Nonnormal and categorical data in structural equation modeling. In G. R. Hancock \& R. O. Mueller (Eds.), Structural Equation Modeling: A Second Course (2014-01991-011; 2nd ed., pp. 439-492). IAP Information Age Publishing.

Homburg, A., Stolberg, A., \& Wagner, U. (2007). Coping with global environmental problems: Development and first validation of scales. Environment and Behavior, 39(6), 754-778. https://doi.org/10.1177/0013916506297215

Hornsey, M. J., Harris, E. A., \& Fielding, K. S. (2018). Relationships among conspiratorial beliefs, conservatism and climate scepticism across nations. Nature Climate Change, 8(7), 614-620. https://doi.org/10.1038/s41558-018-0157-2

Hu, L., \& Bentler, P. M. (1999). Cutoff criteria for fit indexes in covariance structure analysis: Conventional criteria versus new alternatives. Structural Equation Modeling: A Multidisciplinary Journal, 6(1), 1-55. https://doi.org/10.1080/10705519909540118 
IDEOLOGY, NeEDS, GENDER, AND THE SPECTRUM OF DENIAL

Hultman, M., \& Pulé, P. M. (2018). Ecological Masculinities: Theoretical Foundations and Practical Guidance. Routledge. https://doi.org/10.4324/9781315195223

Jost, J. T., \& Banaji, M. R. (1994). The role of stereotyping in system-justification and the production of false consciousness. British Journal of Social Psychology, 33(1), 1-27. https://doi.org/10.1111/j.2044-8309.1994.tb01008.x

Jylhä, K. M., \& Akrami, N. (2015). Social dominance orientation and climate change denial: The role of dominance and system justification. Personality and Individual Differences, 86, 108-111. https://doi.org/10.1016/j.paid.2015.05.041

Jylhä, K. M., Cantal, C., Akrami, N., \& Milfont, T. L. (2016). Denial of anthropogenic climate change: Social dominance orientation helps explain the conservative male effect in Brazil and Sweden. Personality and Individual Differences, 98, 184-187. https://doi.org/10.1016/j.paid.2016.04.020

Kaplan, H., \& Madjar, N. (2015). Autonomous motivation and pro-environmental behaviours among Bedouin students in israel: A self-determination theory perspective. Australian Journal of Environmental Education, 31(2), 223-247. https://doi.org/10.1017/aee.2015.33

Kasser, T., \& Ryan, R. M. (1996). Further examining the American Dream: Differential correlates of intrinsic and extrinsic goals. Personality and Social Psychology Bulletin, 22(3), 280-287. https://doi.org/10.1177/0146167296223006

Leiner, D. J. (2020). SoSci Survey (3.2.21) [Computer software]. https://www.soscisurvey.de McCright, A. M., \& Dunlap, R. E. (2011). Cool dudes: The denial of climate change among conservative white males in the United States. Global Environmental Change, 21(4), 1163-1172. https://doi.org/10.1016/j.gloenvcha.2011.06.003

McLachlan, G. J., \& Peel, D. (2000). Finite Mixture Models (Electronic resources). Wiley. http://ludwig.lub.lu.se/login?url=https://search.ebscohost.com/login.aspx?direct=true \& $\mathrm{db}=$ cat $07147 \mathrm{a} \& \mathrm{AN}=$ lub.6316920\&site $=$ eds-live $\&$ scope $=$ site 
IDEOLOGY, NEEDS, GENDER, AND THE SPECTRUM OF DENIAL

Milfont, T. L., Abrahamse, W., \& MacDonald, E. A. (2021). Scepticism of anthropogenic climate change: Additional evidence for the role of system-justifying ideologies. Personality and Individual Differences, 168, 110237. https://doi.org/10.1016/j.paid.2020.110237

Milfont, T. L., Richter, I., Sibley, C. G., Wilson, M. S., \& Fischer, R. (2013). Environmental consequences of the desire to dominate and be superior. Personality and Social Psychology Bulletin, 39(9), 1127-1138. https://doi.org/10.1177/0146167213490805

Nelson, J. (2020). Petro-masculinity and climate change denial among white, politically conservative American males. International Journal of Applied Psychoanalytic Studies, 17(4), 282-295. https://doi.org/10.1002/aps.1638

Norgaard, K. M. (2006). "People want to protect themselves a little bit": Emotions, denial, and social movement nonparticipation. Sociological Inquiry, 76(3), 372-396.

Norgaard, K. M. (2019). Making sense of the spectrum of climate denial. Critical Policy Studies, 13(4), 437-441. https://doi.org/10.1080/19460171.2019.1671208

Nylund, K. 1., Asparouhov, T., \& Muthen, B. o. (2007). Deciding on the number of classes in latent class analysis and growth mixture modeling: A Monte Carlo simulation study. Structural Equation Modeling, 14(4), 535-569.

Nylund-Gibson, K., \& Choi, A. Y. (2018). Ten frequently asked questions about latent class analysis. Translational Issues in Psychological Science, 4(4), 440-461. https://doi.org/10.1037/tps0000176

Ojala, M. (2012). How do children cope with global climate change? Coping strategies, engagement, and well-being. Journal of Environmental Psychology, 32(3), 225-233. https://doi.org/10.1016/j.jenvp.2012.02.004

Pratto, F., Sidanius, J., Stallworth, L. M., \& Malle, B. F. (1994). Social dominance orientation: A personality variable predicting social and political attitudes. Journal of 
IDEOLOGY, NEEDS, GENDER, AND THE SPECTRUM OF DENIAL

Personality and Social Psychology, 67(4), 741-763. https://doi.org/10.1037/00223514.67.4.741

R Core Team. (2020). R: A Language and Environment for Statistical Computing (4.0.3) [Computer software]. R Foundation for Statistical Computing. https://www.Rproject.org/

Rosenberg, J. M., Beymer, P. N., Anderson, D. J., Van Lissa, C. J., \& Schmidt, J. A. (2018). TidyLPA: An R package to easily carry out latent profile analysis (LPA) using opensource or commercial software. Journal of Open Source Software, 3(30), 978. https://doi.org/10.21105/joss.00978

Rosseel, Y. (2012). \{lavaan\}: An \{R\} Package for Structural Equation Modeling. Journal of Statistical Software, 48(2), 1-36.

Ryan, R. M., \& Deci, E. L. (2017). Self-Determination Theory: Basic Psychological Needs in Motivation, Development, and Wellness. Guilford Press.

Scrucca, L., Fop, M., Murphy, T. B., \& Raftery, A. E. (2016). mclust 5: Clustering, classification and density estimation using Gaussian finite mixture models. The $R$ Journal, 8(1), 289--317. https://doi.org/10.32614/RJ-2016-021

Stanley, S. K., Wilson, M. S., \& Milfont, T. L. (2017). Exploring short-term longitudinal effects of right-wing authoritarianism and social dominance orientation on environmentalism. Personality and Individual Differences, 108, 174-177. https://doi.org/10.1016/j.paid.2016.11.059

Stoll-Kleemann, S., \& O’Riordan, T. (2020). Revisiting the psychology of denial concerning low-carbon behaviors: From moral disengagement to generating social change. Sustainability, 12(3), 935. https://doi.org/10.3390/su12030935

Tein, J.-Y., Coxe, S., \& Cham, H. (2013). Statistical power to detect the correct number of classes in latent profile analysis. Structural Equation Modeling: A Multidisciplinary Journal, 20(4), 640-657. https://doi.org/10.1080/10705511.2013.824781 
IDEOlogy, NeEds, Gender, AND the SPECtrum of Denial

Tröger, J., Wullenkord, M. C., Barthels, C., \& Steller, R. (2021). Can reflective diary-writing increase sufficiency-oriented consumption? A longitudinal intervention addressing the role of basic psychological needs, subjective well-being, and time affluence. Sustainability, 13(9), 4885. https://doi.org/10.3390/su13094885

Vansteenkiste, M., \& Ryan, R. M. (2013). On psychological growth and vulnerability: Basic psychological need satisfaction and need frustration as a unifying principle. Journal of Psychotherapy Integration, 23(3), 263-280. https://doi.org/10.1037/a0032359

Wullenkord, M. C. (2020). Climate change through the lens of Self-Determination Theory: How considering basic psychological needs may bring environmental psychology forward. Umweltpsychologie, 24(2), 110-129.

Wullenkord, M. C., \& Reese, G. (2020). Avoidance, rationalization, and denial: Defensive self-protection in the face of climate change negatively predicts pro-environmental behavior. PsyArXiv. https://doi.org/10.31234/osf.io/uwmcx 